\title{
A NOTE ON SKEW-HOPF FIBRATIONS
}

\author{
MICHAEL E. GAGE
}

\begin{abstract}
Each great circle fibration of the unit 3-sphere in 4-space can be identified with a subset of the Grassmann manifold of oriented 2-planes in 4-space by associating each great circle fiber with the 2-plane it lies in. This Grassmann manifold can be identified with the space $S^{2} \times S^{2}$. H. Gluck and F. Warner, in Great circle fibrations of the three sphere, Duke Math. J. 50 (1983), 107-132, have shown that the subsets of this Grassmann manifold which correspond to great circle fibrations can be interpreted as the graphs of distance decreasing maps from $S^{2}$ and $S^{2}$ and that Hopf fibrations correspond to constant maps.

This note characterizes explicitly the maps which correspond to "skew-Hopf" fibrations: those fibrations of the 3-sphere obtained from Hopf fibrations by applying a linear transformation of 4-space followed by projection of the fibers back to the unit 3-sphere.
\end{abstract}

1. Each great circle fibration of the unit 3-sphere in $\mathbf{R}^{4}$ can be identified with a subset of the Grassmann manifold $G_{2,4}$ of oriented 2-planes in 4-space by associating each great circle fiber with the 2-plane it lies in. It is well known that $G_{2,4}$ can be identified with $S^{2} \times S^{2}$; Gluck and Warner [G-W] have shown that the subsets of $G_{2,4}$ which correspond to great circle fibrations can be interpreted as the graphs of distance decreasing maps from $S^{2}$ to $S^{2}$. In particular, the Hopf fibrations correspond to constant maps.

In this note we describe explicitly all subsets of $G_{2,4}$ which correspond to "skew-Hopf" fibrations; those fibrations of $S^{3}$ obtained from Hopf fibrations by applying a linear transformation of $\mathbf{R}^{4}$ followed by projection of the fibers back to the unit sphere (see $\mathbf{G}-\mathbf{W}]$ ). We use the natural embedding of $G_{2,4}$ into $\Lambda^{2} \mathbf{R}^{4}$ to show that all such subsets are the intersections of 4-dimensional linear subspaces of $\Lambda^{2} \mathbf{R}^{4}$ with $G_{2,4}$. We define a symmetric, nondegenerate, nondefinite form $Q$ and show that a 4-dimensional linear subspace intersects $G_{2,4}$ in a "skew-Hopf subset" if and only if $Q$ restricted to the subspace has signature $(+,-,-,-)$ or $(+,+,+,-)$. The proof consists of showing that the Hopf fibration is the intersection of a specific linear 4-dimensional subspace of $\Lambda^{2} \mathbf{R}^{4}$ and determining the orbit of this subspace under the action induced on $\Lambda^{2} \mathbf{R}^{4}$ by the affine linear group of $\mathbf{R}^{4}$. In addition, we obtain a geometric picture of the distance decreasing maps from $S^{2}$ to $S^{2}$ which correspond to skew-Hopf fibrations. It follows easily from this picture that such maps have convex images.

Received by the editors November 4, 1983 and, in revised form, February 8, 1984.

1980 Mathematics Subject Classification. Primary 55R25, 14M15; Secondary 51N30, 14L35.

Key words and phrases. Hopf fibration, great circle fibration, Grassmann manifold. 
2. Notation and preliminaries. If $e_{1}, \ldots, e_{4}$ is an orthonormal basis for $\mathbf{R}^{4}$, then $\left\{e_{i} \wedge e_{j} \mid 1 \leqslant i<j \leqslant 4\right\}$ forms an orthonormal basis for the space of bivectors $\Lambda^{2} \mathbf{R}^{4}$ and specifies an isomorphism of $\Lambda^{2} \mathbf{R}^{4}$ with $\mathbf{R}^{6}$. In addition to the usual inner product induced on $\Lambda^{2} \mathbf{R}^{4}$, there is a second bilinear form $Q$ obtained from the map $\Lambda^{2} \mathbf{R}^{4} \times \Lambda^{2} \mathbf{R}^{4} \rightarrow \Lambda^{4} \mathbf{R}^{4} \rightarrow \mathbf{R}$ which takes the wedge product of two bivectors of $\mathbf{R}^{4}$ to a scalar times the volume form of $\mathbf{R}^{4}$. This bilinear form is symmetric because the wedge product of bivectors is commutative.

It is easily checked that the Plücker basis

$$
\begin{array}{ll}
i_{1}=\frac{e_{1} \wedge e_{2}+e_{3} \wedge e_{4}}{\sqrt{2}}, & i_{4}=\frac{e_{1} \wedge e_{2}-e_{3} \wedge e_{4}}{\sqrt{2}}, \\
i_{2}=\frac{e_{1} \wedge e_{3}-e_{2} \wedge e_{4}}{\sqrt{2}}, & i_{5}=\frac{e_{1} \wedge e_{3}+e_{2} \wedge e_{4}}{\sqrt{2}}, \\
i_{3}=\frac{e_{1} \wedge e_{4}+e_{2} \wedge e_{3}}{\sqrt{2}}, & i_{6}=\frac{e_{1} \wedge e_{4}-e_{2} \wedge e_{3}}{\sqrt{2}},
\end{array}
$$

is an orthonormal basis which diagonalizes the matrix representing $Q$. On the subspace $\Lambda_{+}^{2} \mathbf{R}^{4}$ spanned by $i_{1}, i_{2}$ and $i_{3}, Q$ has eigenvalue +1 ; on the subspace $\Lambda_{-}^{2} \mathbf{R}^{4}$ spanned by $i_{4}, i_{5}$ and $i_{6}$, the eigenvalue is -1 .

Further calculation shows that a bivector $\omega$ in $\Lambda^{2} \mathbf{R}^{4}$ can be written as the wedge product of two vectors in $\mathbf{R}^{4}$ if and only if $Q(\omega, \omega)=0$; these are the simple bivectors of $\Lambda^{2} \mathbf{R}^{4}$. Identifying the oriented 2-planes of $\mathbf{R}^{4}$ with the wedge product of an orthonormal basis which spans it (and possesses the same orientation) yields a one-to-one correspondence between $G_{2,4}$ and the simple bivectors of $\Lambda^{2} \mathbf{R}^{4}$ which have unit length. The simple bivectors of unit length form the set

$$
S_{+}^{2}(1 / \sqrt{2}) \times S_{-}^{2}(1 / \sqrt{2}) \subseteq \Lambda_{+}^{2} \mathbf{R}^{4} \oplus \Lambda_{-}^{2} \mathbf{R}^{4} .
$$

It is convenient to represent elements of $\Lambda^{2} \mathbf{R}^{4}$ as a pair of vectors in $\mathbf{R}^{3}$; that is, $\omega \equiv\left(\omega^{+}, \omega^{-}\right)$, where $\omega^{+}$is the projection of $\omega$ onto $\Lambda_{+}^{2} \mathbf{R}^{4}$ and $\omega^{-}$is the projection onto $\Lambda_{-}^{2} \mathbf{R}^{4}$. In this notation $Q(\omega, \eta)=\left\langle\omega^{+}, \eta^{+}\right\rangle-\left\langle\omega^{-}, \eta^{-}\right\rangle$and $\langle\omega, \eta\rangle$ $=\left\langle\omega^{+}, \eta^{+}\right\rangle+\left\langle\omega^{-}, \eta^{-}\right\rangle$. The star operator $\left(^{*}\right)$ is an involution defined by ${ }^{*} \omega=$ ${ }^{*}\left(\omega^{+}, \omega^{-}\right)=\left(\omega^{+},-\omega^{-}\right)$which satisfies $Q(\omega, \eta)=\left\langle\omega,{ }^{*} \eta\right\rangle$.

It will also be useful to understand the action of elements of the special orthogonal groups $\operatorname{SO}\left(\mathbf{R}^{4}\right)$ and the special linear groups $\operatorname{SL}\left(\mathbf{R}^{4}\right)$ on $\Lambda^{2} \mathbf{R}^{4}$. Since elements of $\operatorname{SO}\left(\mathbf{R}^{4}\right)$ preserve both the inner product on $\Lambda^{2} \mathbf{R}^{4}$ and the bilinear form $Q$, one can show by direct calculation that the action of $\operatorname{SO}\left(\mathbf{R}^{4}\right)$ on $\Lambda^{2} \mathbf{R}^{4}$ is to rotate each eigenspace of $Q$ independently and that the map $\operatorname{SO}\left(\mathbf{R}^{4}\right) \rightarrow \operatorname{SO}\left(\mathbf{R}^{3}\right) \times \operatorname{SO}\left(\mathbf{R}^{3}\right)$ is a double covering. For example, rotation in the $e_{1}, e_{2}$ and $e_{3}, e_{4}$ planes simultaneously by an angle $\theta$ induces a rotation in the $i_{2}, i_{3}$ plane of $\Lambda^{2} \mathbf{R}^{4}$ through an angle of $2 \theta$ and leaves the other basis vectors fixed.

Elements of $\operatorname{SL}\left(\mathbf{R}^{4}\right)$ preserve the bilinear form $Q$, but not necessarily the inner product. The element of $\operatorname{SL}\left(\mathbf{R}^{4}\right)$ which multiplies the $e_{1}, e_{2}$ plane by $e^{\phi}$ and the $e_{3}, e_{4}$ plane by $e^{-\phi}$ induces an action on $\Lambda^{2} \mathbf{R}^{4}$ which sends $i_{1}$ to $(\cosh 2 \phi) i_{1}+(\sinh 2 \phi) i_{4}$ and $i_{4}$ to $(\sinh 2 \phi) i_{1}+(\cosh 2 \phi) i_{4}$, while leaving the other vectors fixed. These facts can be verified by direct calculation and will be used in the proof of Lemma 2 . 
LEMMA 1. The Hopf fibration corresponds to the subset

$$
\left\{\left(i_{1} / \sqrt{2}, v\right) \in S_{+}^{2}(1 / \sqrt{2}) \times S_{-}^{2}(1 / \sqrt{2}) \mid v \in S_{-}^{2}\right\} \subseteq G_{2,4} .
$$

This subset is topologically \{point $\times S^{2}$ and can be considered as the graph of the constant map from $S_{-}^{2}$ to $S_{+}^{2}$. It is one component of the intersection with $G_{2,4}$ of the 4-dimensional subspace spanned by $i_{1}, i_{4}, i_{5}, i_{6}$. The other component represents the same fibration with the orientation of the fibers reversed.

Proof. We identify $\mathbf{R}^{4}$ with $\mathbf{C}^{2}$. The unit sphere is the set $\left\{\left.\left(z_{1}, z_{2}\right) \in \mathbf{C}^{2}|| z_{1}\right|^{2}+\right.$ $\left.\left|z_{2}\right|^{2}=1\right\}$ and the great circle fibers of the Hopf fibration are the orbits $\left\{\left(z_{1} e^{i \theta}, z_{2} e^{i \theta}\right) \mid \theta \in[0,2 \pi)\right\}$. Multiplication of $\left(z_{1}, z_{2}\right)$ by $e^{i \theta}$ in this way is equivalent to rotating in the $e_{1}, e_{2}$ and $e_{3}, e_{4}$ planes simultaneously by an angle $\theta$. All 2-planes left fixed by this action correspond to fibers of the Hopf fibration and to those points in $G_{2,4}$ which remain fixed under the induced action. This set of fixed points is the intersection of $G_{2,4}$ with the linear subspace spanned by $i_{1}, i_{4}, i_{5}$, and $i_{6}$ as can be found by direct calculation. Topologically it is $S^{0} \times S^{2}$. Each component corresponds to a different orientation on the great circle fibers. (See [G-W] for another derivation of this.) When restricted to this subspace $Q$ has signature $(+,-,-,-)$.

LEMMA 2. The action of an element $g \in \mathrm{SL}\left(\mathbf{R}^{4}\right)$ induces a linear action $\hat{g}$ on $\Lambda^{2} \mathbf{R}^{4}$ which preserves the form $Q$. Thus $\hat{g}$, acting on one of the 4-dimensional linear subspaces $L_{4}$ of $\Lambda^{2} \mathbf{R}^{4}$, yields a subspace, $\hat{g}\left(L_{4}\right)$ such that $\left.Q\right|_{L_{4}}$ and $\left.Q\right|_{\hat{g}\left(L_{4}\right)}$ have the same signature. Conversely, if $\left.Q\right|_{L_{4}}$ and $\left.Q\right|_{L_{4}^{\prime}}$ have the same signature, then there is an element $g$ of $\mathrm{SL}\left(\mathbf{R}^{4}\right)$ which induces an action $\hat{g}$ which takes $L_{4}$ to $L_{4}^{\prime}$.

Proof. For simple bivectors $\omega=v_{1} \wedge v_{2}$ and $\eta=v_{3} \wedge v_{4}$ and $g \in \operatorname{SL}\left(\mathbf{R}^{4}\right)$, we have, since det $g=1$,

$$
\begin{aligned}
Q(\hat{g}(\omega), \hat{g}(\eta)) e_{1} \wedge e_{2} \wedge e_{3} \wedge e_{4} & =g\left(v_{1}\right) \wedge g\left(v_{2}\right) \wedge g\left(v_{3}\right) \wedge g\left(v_{4}\right) \\
& =(\operatorname{det} g) v_{1} \wedge v_{2} \wedge v_{3} \wedge v_{4} \\
& =Q(\omega, \eta) e_{1} \wedge e_{2} \wedge e_{3} \wedge e_{4} .
\end{aligned}
$$

The linearity properties of $Q$ and $\hat{g}$ insure that this holds for linear combinations of simple bivectors also. For the second statement one can show that the action of $\operatorname{SL}\left(\mathbf{R}^{4}\right)$ on $G_{2,4}$ is one component of the automorphism group of $Q$ and, hence, by Witt's theorem [A, p. 121] there is an element of $\operatorname{SL}\left(\mathbf{R}^{4}\right)$ which takes any $L_{4}$ of $G_{2,4}$ to any other $L_{4}^{\prime}$ with the same signature.

To see this directly, without Witt's theorem, we construct adapted frames for $L_{4}$ and $L_{4}^{\prime}$ and construct the element of $\operatorname{SL}\left(\mathbf{R}^{4}\right)$ which takes one frame to the other:

For any 4-dimensional linear subspace $L_{4}$ we can find elements $\eta_{1}$ and $\eta_{2}$ of $\Lambda^{2} \mathbf{R}^{4}$ which satisfy

(1) $\left\langle\eta_{i}, \eta_{j}\right\rangle=\delta_{i j}, i, j=1,2$,

(2) $Q\left(\eta_{1}, \eta_{2}\right)=0$

(3) $Q\left(\eta_{i}, \lambda\right)=0$ for all $\lambda \in L_{4}$ and $i=1,2$. 
The 2-dimensional subspace spanned by $\eta_{1}, \eta_{2}$ is $Q$-orthogonal to $L_{4}$ by (3). These subspaces can be classified by the signature of the restriction of $Q$ to the subspaces. Furthermore, since $L_{4}$ and the 2-dimensional subspace are $Q$ orthogonal, one can extend $\eta_{1}, \eta_{2}$ to a basis of $\Lambda^{2} \mathbf{R}^{4}$ which diagonalizes $Q$, and therefore the signature of $Q$ on the 2-dimensional space determines the signature of $Q$ on the 4-dimensional space and vice versa.

The construction of $\eta_{1}$ and $\eta_{2}$ satisfying (1)-(3) proceeds as follows: Choose orthonormal vectors $\omega_{1}, \omega_{2} \in \Lambda^{2} \mathbf{R}^{4}$ perpendicular to $L_{4}$ (i.e., $\left\langle\omega_{1}, \lambda\right\rangle=\left\langle\omega_{2}, \lambda\right\rangle=0$ for all $\left.\lambda \in L_{4}\right)$. By a rotation in the $\omega_{1}, \omega_{2}$ plane, we obtain a new basis $\tilde{\omega}_{1}, \tilde{\omega}_{2}$ which diagonalizes $Q$ (i.e., $Q\left(\tilde{\omega}_{1}, \tilde{\omega}_{2}\right)=0$ ). Let $\eta_{1}={ }^{*} \tilde{\omega}_{1}$ and $\eta_{2}={ }^{*} \tilde{\omega}_{2}$ and use the fact that $\left\langle{ }^{*} \omega,{ }^{*} \eta\right\rangle=\langle\omega, \eta\rangle$ and $Q(\omega, \eta)=\left\langle\omega,{ }^{*} \eta\right\rangle$ to verify (1)-(3).

We now construct an action of $\operatorname{SL}\left(\mathbf{R}^{4}\right)$ which moves the vector $\eta_{1}, \eta_{2}$ to a multiple of $i_{1}, i_{2}$ provided $Q\left(\eta_{1}, \eta_{1}\right)>0$ and $Q\left(\eta_{2}, \eta_{2}\right)>0$.

We represent $\eta_{1}$ as $\left(\eta_{1}^{+}, \eta_{1}^{-}\right)$and $\eta_{2}$ as $\left(\eta_{2}^{+}, \eta_{2}^{-}\right)$. There is an element of $g$ of $\operatorname{SO}\left(\mathbf{R}^{4}\right)$ which rotates $\Lambda^{2} \mathbf{R}^{4}$ so that $\eta_{1}^{+}$and $\eta_{2}^{+}$are parallel with $i_{1}$ and $i_{2}$, respectively, and which simultaneously rotates $\Lambda_{-}^{2} \mathbf{R}^{4}$ so that $\eta_{1}^{-}$and $\eta_{2}^{-}$are aligned with $i_{4}$ and $i_{5}$. (Note that $\left\langle\eta_{1}^{+}, \eta_{2}^{+}\right\rangle=\left\langle\eta_{1}^{-}, \eta_{2}^{-}\right\rangle=0$ as a consequence of (1) and (3).) Direct computation shows that the diagonal matrix with entries $\left(e^{\phi}, e^{\phi}, e^{-\phi}, e^{-\phi}\right)$ represents an element of $\operatorname{SL}\left(\mathbf{R}^{4}\right)$ which takes $g\left(\eta_{1}\right)=a i_{1}+b i_{4}$ to $(a \cosh 2 \phi+b \sinh 2 \phi) i_{1}+(a \sinh 2 \phi$ $+b \cosh 2 \phi) i_{4}$ and leaves $g\left(\eta_{2}\right)$ fixed (see $\left.\$ 2\right)$. Since $Q\left(\eta_{1}, \eta_{1}\right)=Q\left(g\left(\eta_{1}\right), g\left(\eta_{1}\right)\right)=$ $a^{2}-b^{2}>0$, it is possible to find a $\phi$ which makes the coefficient of $i_{4}$ zero. Similarly, we can find an element of $\operatorname{SL}\left(\mathbf{R}^{4}\right)$ which takes $g\left(\eta_{2}\right)$ to a multiple of $i_{2}$. The composition of these three transformations is an element $g$ of $\operatorname{SL}\left(\mathbf{R}^{4}\right)$ whose action on $\Lambda^{2} \mathbf{R}^{4}$ takes $\eta_{1}$ to a multiple of $i_{1}$ and $\eta_{2}$ to a multiple of $i_{2}$.

Since this transformation preserves $Q$ it takes $L_{4}$ to the space spanned by $i_{3}, i_{4}, i_{5}$, and $i_{6}$.

For any other $L_{4}^{\prime}$ with the same signature there is an action $g^{\prime}$ of $\operatorname{SL}\left(\mathbf{R}^{4}\right)$ which also takes $L_{4}^{\prime}$ to the space spanned by $i_{3}, i_{4}, i_{5}$ and $i_{6}$ and, therefore, $\left(g^{\prime}\right)^{-1} \circ g$ takes $L_{4}$ to $L_{4}^{\prime}$. This completes the proof when $\left.Q\right|_{L_{4}}$ has signature $(+,-,-,-)$; similar arguments handle the other possible signatures of $\left.Q\right|_{L_{4}}$.

From Lemmas 1 and 2 we immediately deduce:

Proposition 3. There is a one-to-one correspondence between skew-Hopf fibrations and those 4-dimensional subspaces $L_{4}$ of $\Lambda^{2} \mathbf{R}^{4}$ such that $\left.Q\right|_{L_{4}}$ has signature $(+,+,+,-)$ or $(+,-,-,-)$.

Proof. The element $g \in \operatorname{SL}\left(\mathbf{R}^{4}\right)$ which takes the Hopf fibration in Lemma 1 to a skew Hopf fibration also takes the 4-plane spanned by $i_{1}, i_{4}, i_{5}, i_{6}$ to a 4-dimensional subspace whose intersection with $G_{2,4}$ corresponds to the skew-Hopf fibration. When $Q$ is restricted to this subspace it has signature $(+,-,-,-)$. On the other hand, given any 4-dimensional subspace with this signature, there is a $g$ in $\operatorname{SL}\left(\mathbf{R}^{4}\right)$ such that $\hat{g}$ takes $\left\{i_{1}, i_{4}, i_{5}, i_{6}\right\}$ to the given subspace (Lemma 2) and $g$ takes the standard Hopf fibration to a corresponding skew Hopf fibration.

The reflection which takes $e_{4}$ to $-e_{4}$ takes the standard Hopf fibration to one with reverse screw sense and takes the corresponding 4-plane to one spanned by $i_{1}, i_{2}, i_{3}, i_{4}$ which has signature $(+,+,+,-)$. This completes the proof. 
We know from [G-W] that all great circle fibrations of $S^{3}$ correspond to the graph of distance decreasing maps from $S^{2}$ to $S^{2}$. The next task is to describe explicitly the maps corresponding to skew-Hopf fibrations. Choosing the appropriate coordinates in $\Lambda^{2} \mathbf{R}^{4}$ makes this easy.

Assume that $L_{4}$ is a fixed 4-dimensional subspace which corresponds to a skew-Hopf fibration and for which $\left.Q\right|_{L_{4}}$ has signature $(+,-,-,-)$. Choose the unit vectors $\eta_{1}$ and $\eta_{2}$ of $\Lambda^{2} \mathbf{R}^{4}$ which span the space $Q$-orthogonal to $L_{4}$ and which satisfy (1)-(3). In terms of the decomposition $\Lambda^{2} \mathbf{R}^{4}=\Lambda_{+}^{2} \mathbf{R}^{4} \oplus \Lambda_{-}^{2} \mathbf{R}^{4}=\mathbf{R}^{3} \oplus \mathbf{R}^{3}$, we write $\eta_{1}=\left(\eta_{1}^{+}, \eta_{1}^{-}\right)$and $\eta_{2}=\left(\eta_{2}^{+}, \eta_{2}^{-}\right)$. From (1) and (2) we have

$$
\left\langle\eta_{1}, \eta_{2}\right\rangle=\left\langle\eta_{1}^{+}, \eta_{2}^{+}\right\rangle+\left\langle\eta_{1}^{-}, \eta_{2}^{-}\right\rangle=0
$$

and

$$
Q\left(\eta_{1}, \eta_{2}\right)=\left\langle\eta_{1}^{+}, \eta_{2}^{+}\right\rangle-\left\langle\eta_{1}^{-}, \eta_{2}^{-}\right\rangle=0,
$$

and therefore $\eta_{1}^{+}, \eta_{2}^{+}, \eta_{1}^{-}$and $\eta_{2}^{-}$are orthogonal (but not necessarily of unit length). We can choose a coordinate system $\left(x_{1}, x_{2}, x_{3}, y_{1}, y_{2}, y_{3}\right)$ of $\Lambda_{+}^{2} \mathbf{R}^{4} \oplus \Lambda_{-}^{2} \mathbf{R}^{4}$ so that $\eta_{i}^{+}$is parallel to the $x_{i}$-axis and $\eta_{i}^{-}$is parallel to the $y_{i}$-axis for $i=1,2 . \eta_{2}^{+}$would be represented in coordinates by $\left(0,\left|\eta_{2}^{+}\right|, 0,0,0,0\right)$, where $\left|\eta_{2}^{+}\right|$is the length of $\eta_{2}^{+}$.

In terms of these coordinates we can express an arbitrary element $\omega=$ $\left(x_{1}, x_{2}, x_{3}, y_{1}, y_{2}, y_{3}\right)$ and can write the following conditions in coordinate terms:

If $\omega$ is in $G_{2,4}$ then

$$
0=Q(\omega, \omega)=x_{1}^{2}+x_{2}^{2}+x_{3}^{2}-y_{1}^{2}-y_{2}^{2}-y_{3}^{2}
$$

and

$$
1=\langle\omega, \omega\rangle=x_{1}^{2}+x_{2}^{2}+x_{3}^{2}+y_{1}^{2}+y_{2}^{2}+y_{3}^{2} .
$$

If $\omega$ is also in $L_{4}$ and $Q$-orthogonal to $\eta_{1}$ and $\eta_{2}$, then

$$
0=Q\left(\omega, \eta_{i}\right)=x_{i}\left|\eta_{i}^{+}\right|-y_{i}\left|\eta_{i}^{-}\right|, \quad i=1,2 .
$$

The condition that $Q\left(\eta_{i}, \eta_{i}\right)>0$ becomes

$$
0<Q\left(\eta_{i}, \eta_{i}\right)=\left|\eta_{i}^{+}\right|^{2}-\left|\eta_{i}^{-}\right|^{2}, \quad i=1,2 .
$$

We rewrite (7) as

$$
x_{1}=\left(\left|\eta_{1}^{-}\right| /\left|\eta_{1}^{+}\right|\right) y_{1}
$$

and

$$
x_{2}=\left(\left|\eta_{2}^{-}\right| /\left|\eta_{2}^{+}\right|\right) y_{2},
$$

and from (5) and (6) we also have

$$
x_{3}=+\sqrt{1 / 2-x_{1}^{2}-x_{2}^{2}} .
$$

Thus each element $\omega$ in the plane $L_{4}$ is in the graph of the function defined from $S^{2}(1 / \sqrt{2})$ to $S^{2}(1 / \sqrt{2})$ by (9)-(11), or is in the graph of the function defined by changing the sign in front of the radical sign in (11). We can describe these equations geometrically as follows: 
Proposition 4. Each skew-Hopf fibration corresponds to a distance decreasing map from $S^{2}(1 / \sqrt{2}) \rightarrow S^{2}(1 / \sqrt{2})$ which can be decomposed as: (a) an orthogonal projection $P$ to a plane through the center of the sphere; followed by (b) a distance decreasing linear map $A$ from one 2-plane to another; and finally (c) inverse projection onto $S^{2}(1 / \sqrt{2})$.

Proof. The orthogonal projection in the coordinate system above is onto the $\left(y_{1}, y_{2}\right)$-plane. Equations (9) and (10) define the linear map and are distance decreasing because $\left|\eta_{i}^{-}\right| /\left|\eta_{i}^{+}\right|<1$ from (8). The choice of inverse projection $P^{-1}$ determines the sign in (11).

Proposition 5. The maps $S^{2} \rightarrow S^{2}$ which correspond to skew-Hopf fibrations have a convex image.

Proof. All such maps can be written in the form $P^{-1} \circ A \circ P$ according to Proposition 4. The image of $A \circ P$ is the interior of an ellipse $E$ which lies inside the unit disk. It remains to show that $P^{-1}$ maps an ellipse to a convex region in the sphere. Let $x$ and $y$ be two points in the image of $P^{-1} \circ A \circ P$ and let $\gamma$ represent the great circle passing through these points. Considering the projection onto the plane we see that $P(\gamma)$ is an ellipse inscribed in the unit circle which intersects the ellipse $E$ in at most four points.

The portion of $\gamma$ which lies in the northern hemisphere projects to a half-ellipse whose endpoints lie on the unit circle and which, by symmetry, intersects the boundary of $E$ only twice. The segment from $P(x)$ to $P(y)$ must lie in the interior of $E$. It follows that the distance minimizing geodesic from $x$ to $y$ lies inside $\operatorname{Im}\left(P^{-1} \circ A \circ P\right)$ and the proposition is proved.

\section{REFERENCES}

[A] E. Artin, Geometric algebra, Interscience, New York, 1957.

[G-W] H. Gluck and F. Warner, Great circle fibrations of the three sphere, Duke Math J. 50 (1983), $107-132$.

Department of Mathematics, Case Western Reserve University, Cleveland, Ohio 44106

Current address: Department of Mathematics, University of Rochester, Rochester, New York 14627 